\title{
ANALYSIS AND SYNTHESIS OF CHAOTIC CIRCUITS USING MEMRISTOR PROPERTIES
}

\author{
Zdeněk Hruboš ${ }^{*}$ - Tomáš Gotthans ${ }^{*, * *}$
}

\begin{abstract}
This paper provides an innovative practical realization of a memristor based chaotic circuit. The first part discusses the mathematical analysis of the proposed system, including calculation of an eigenvalues, bifurcation diagram and largest Lyapunov exponents. Another parts deal with circuitry realization and the influence of parasitic properties of active elements. The circuit simulations obtained by PSpice environment and the practical measurement results on a breadboard are presented in the last part of this paper. The main aim of this work is an innovative realization of the memristor based chaotic circuit with one type of energy-storage element (linear passive capacitor) and with simpler construction in comparison to other circuits. The next contribution consists in verification of designed circuit with respect to influence of parasitic properties of active elements to chaos destruction.

Keywords: differential equations, nonlinear, eigenvalues, Lyapunov exponent, bifurcation, OTA, parasitic, plane projection
\end{abstract}

\section{INTRODUCTION}

Forty years ago today, the memristor was postulated as the fourth circuit element by Leon O. Chua $[1,2]$. In a seminal paper [3], which appeared on 1 May 2008 issue of Nature, a team led by R. Stanley Williams from the Hewlett-Packard Company announced the fabrication of a passive solid-state two-terminal device called the memristor. It thus took its place along side the rest of the more familiar circuit elements such as the resistor, capacitor and inductor. The common thread that binds these four elements together as the four basic elements of circuit theory is the fact that the characteristics of these elements relate the four variables in electrical engineering (voltage, current, flux and charge) intimately [5]. The memristor element, with memristance $\mathrm{M}$, provides a functional relation between charge and flux, $d \varphi=M d q$ [3]. Last five years, the research of circuits containing memristor is becoming a hot topic in the circuit theory and chaos. Over this period, chaotic attractor has been observed in many autonomous memristor based chaotic circuits and many authors papers uses a passive nonlinearity based on memristor [4-13]. Chaotic oscillator containing memristor still attracts attention. One of the first memristor based chaotic circuit was proposed by Itoh and Chua in 2008 [4]. In this case and many others, memristor represents nonlinear function (eg Chua's diode) and together with other elements (eg resistors, capacitors and inductors) is possible to realize a simple chaotic oscillators $[4,5,8,11,12,14]$. Many others authors also deals with modeling and realization of memristor [15-21]. Nevertheless, this paper is not concentrated on memristor elements realization itself. Its nonlinear and dynamical properties are used for the realization of a simple chaotic system, where the memristor function is integral part of circuit.
In this paper is presented memristor based chaotic circuit synthesis based on mathematical model published by Muthuswamy and Chua [6].Muthuswamy and Chua used the classical operational amplifier as the basic building block for circuit synthesis. Compared to them we used an operational transconductance amplifier with a single output (OTA) and multiple output (MOTA). This step led to the simplify the overall circuit structure and we saved one active element. The operational transconductance amplifier is an amplifier whose differential input voltage produces an output current. Thus, it is a voltage controlled current source (VCCS). There is usually an additional input for a current to control the amplifier's transconductance. Single input OTA has only one high-impedance node, in contrast to conventional operational amplifiers. This makes the OTA an excellent device candidate for high-frequency and voltage (or current) programmable analog basic building blocks. Recently, several authors have been successfully using the OTA as the main active element in a continuous-time active filters [26] and especially for the nonlinear chaotic systems realizations [22][24]. In 1989 Sanchez-Sinencio et al. [25] showed that the OTA, as the active element in basic building blocks, can be efficiently used for nonlinear continuous-time function synthesis. Our paper also deals with authentication how to simply realize natural nonlinear chaotic system by electronic equivalent employing OTA elements and analysis of input and output parasitic properties of used active elements. It is very interesting thing, because chaos systems are very sensitive on initial condition and properties of circuit elements. From this point of view is very important to deal with question, whether parasitic properties are not critical for system function. These parasitic elements can cause significant problems in the state space and chaos destruction in the worst case.

* Deptartment of Radio Electronics, Brno University of Technology, Purkyňova 118, 61200 Brno, Czech Republic, ** Universite Paris-Est, ESYCOM, ESIEE Paris, BP99, 93162 Noisy Le Grand Cedex, France, xhrubo00@stud.feec.vutbr.cz, gotthant@esiee.fr 


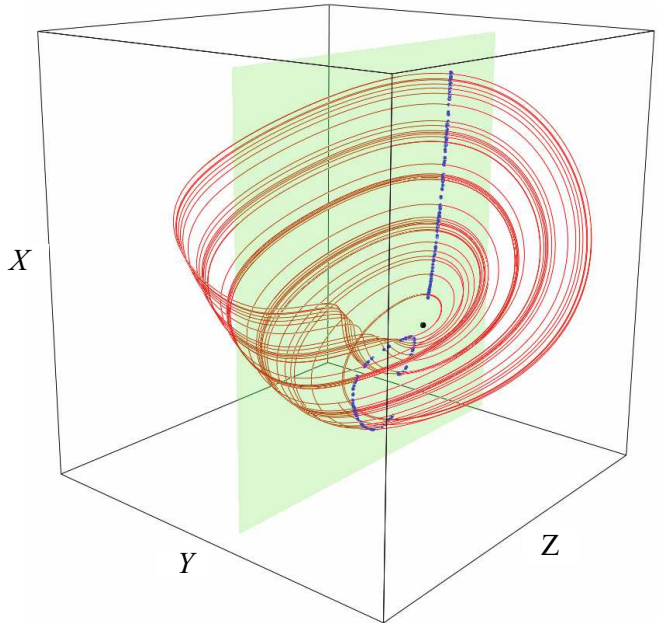

Fig. 1. Numerical simulation in MathCAD and a Poincare section (blue dots) which is formed by $x-z$ plane sliced at $y=0$ (green surface)

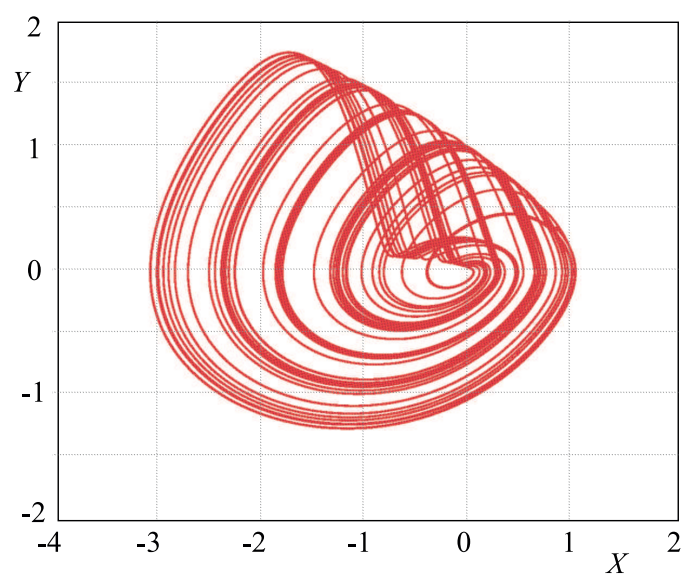

Fig. 2. Plot of $x(t)$ versus $y(t)$ plane projection of the chaotic attractor generated by (1) - numerical solution

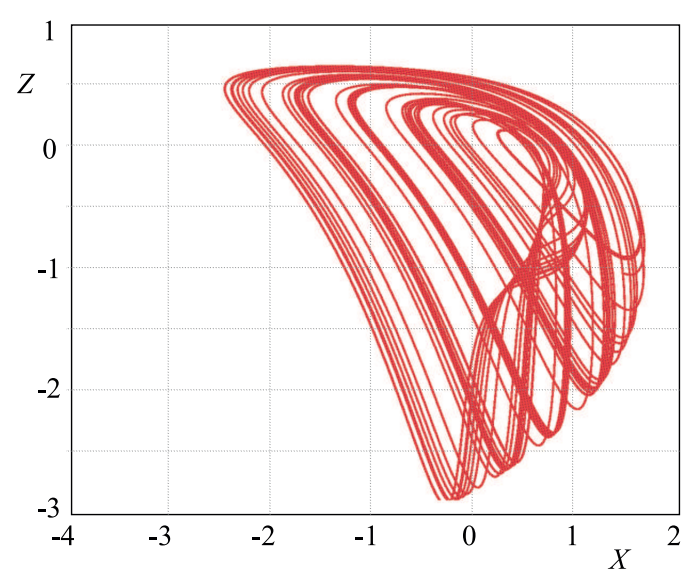

Fig. 3. Plot of $x(t)$ versus $z(t)$ plane projection of the chaotic attractor generated by (1) - numerical solution

This paper is organized in the follows lines: we begin by discussing a fundamental mathematical model of the memristor based chaotic circuit. The next part give an overview of a numerical simulation together with numeri- cal computation of the Lyapunov exponents and bifurcation diagram. In the following part is discussed circuitry implementation and the analysis of influence of parasitic properties of active elements to realized circuit. In the last part are shown simulation and experimental results leading to a chaos.

\section{MATHEMATICAL ANALYSIS}

Consider the three-element circuit with the memristor properties [6]. The equations for the memristor based chaotic circuit are described by set of follows an ordinary differential equations (ODE)

$$
\begin{aligned}
& \dot{x}=y, \\
& \dot{y}=-\frac{1}{3} x+\frac{1}{2} y-\frac{1}{2} z^{2} y, \\
& \dot{z}=-y-\alpha z+z y,
\end{aligned}
$$

where parameter $\alpha=0.6$ can be considered as a bifurcation parameter. Please note that our memristor based chaotic circuit is based on a memristive device defined by Chua and Kang in 1976 [2] and not the ideal memristor defined by Chua in 1971 [1]. System behavior is dependent on the value of many parameters and includes various types of solutions (periodic, quasi periodic or chaos). Figure 1 shows a $3 \mathrm{D}$ plot of the attractor obtained by the numerical simulation of (1) (initial conditions: $x(0)=0.1$, $y(0)=0, z(0)=0.1$ ) by a program MathCAD. Blue dots in Fig. 1 represents a Poincare section (the intersection of a periodic orbit in the state space of a continuous dynamical system with a certain lower dimensional subspace transversal to the flow of the system). Embedded RungeKutta fourth order method in a MathCAD environment was used for a numerical integration of differential equation system. The parameters of the numerical integration are consistent. Time interval was $t(0,500)$ and step was $\Delta t=10^{-2}$. The chaotic attractors projections associated with the numerical integration of the mathematical model are shown in Figs. 2 and 3. The very important characteristic of the chaos system is, that the involvement of system is very sensitive to an initial conditions and the behavior is hard to predict in a long time range [29-32]. Figure 4 illustrates system sensitivity to the changes in initial conditions. Difference between a reference trajectory and a perturbation trajectory is for $I C_{1}=\left(\begin{array}{lll}0.1 & 0 & 0.1\end{array}\right)^{T}$ and $I C_{2}=\left(\begin{array}{lll}0.11 & 0 & 0.11\end{array}\right)^{T}$. We can see that two close solutions diverge from each other and we can expect general validity of this claim.

The position of equilibria (critical) point is independent on the system parameters and is located at $f=$ $[0,0,0]^{T}$ (black dot in Fig. 1). Investigation of vinicity around point $f$ is given by

$$
\operatorname{det}(\lambda \mathbf{I}-\mathbf{J})=0 .
$$


The Jacobian matrix of this system is

$$
\mathbf{J}=\left(\begin{array}{ccc}
0 & 1 & 0 \\
-\frac{1}{3} & -\frac{1}{2} z^{2}+\frac{1}{2} & -y z \\
0 & z-1 & y-0.6
\end{array}\right)
$$

and leads to a characteristic polynomial. For critical point $f$ is following

$$
\begin{aligned}
\operatorname{det}(\lambda \mathbf{I}-\mathbf{J})= & \left|\begin{array}{ccc}
\lambda & -1 & 0 \\
\frac{1}{3} & \lambda-0.5 & 0 \\
0 & 1 & \lambda+0.6
\end{array}\right|= \\
& \lambda^{3}+0.1 \lambda^{2}+0.033 \lambda+0.2=0 .
\end{aligned}
$$
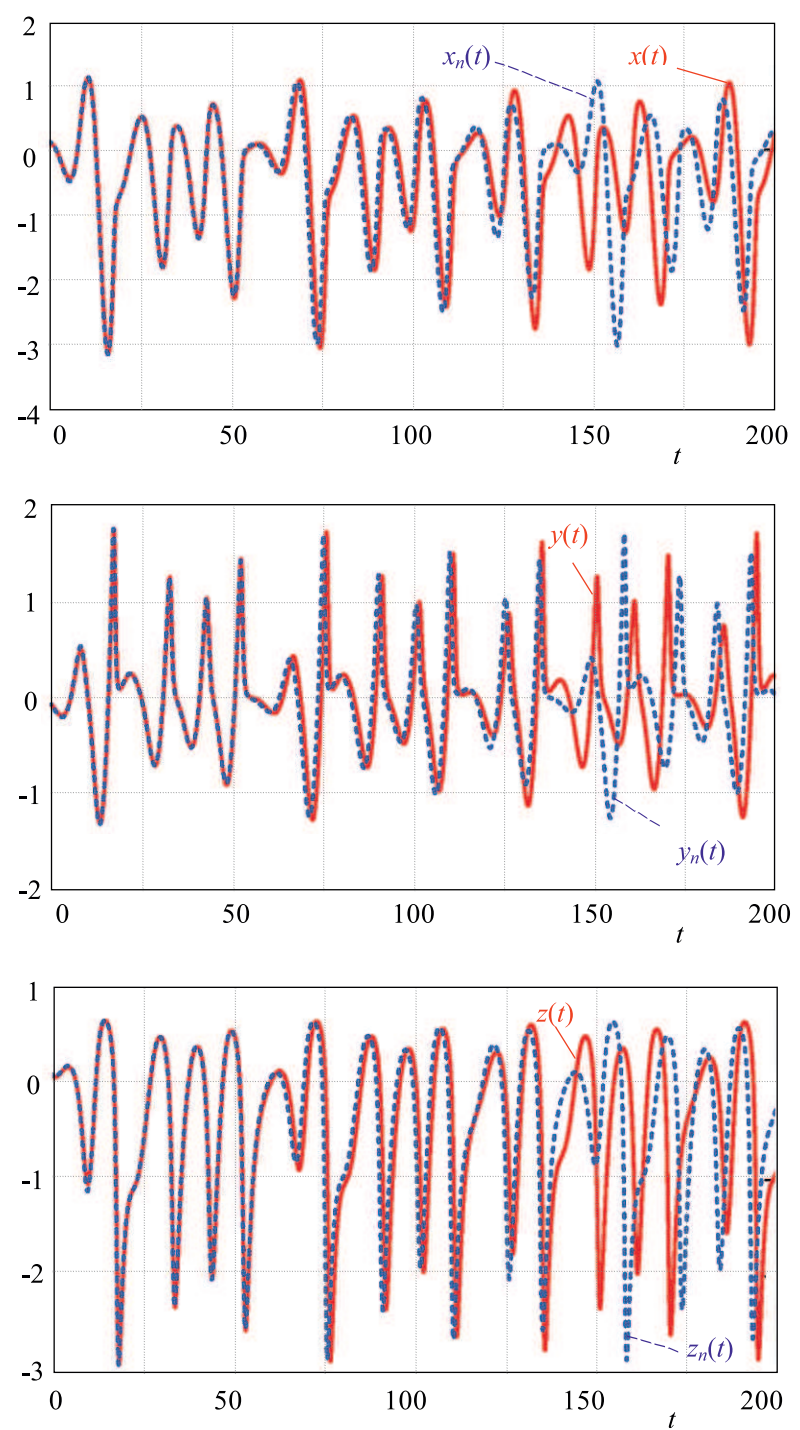

Fig. 4. Plot of $x(t)$ versus $z(t)$ plane projection of the chaotic attractor generated by (1) - numerical solution

The local behavior of the system near the origin is uniquely determined by the eigenvalues:

$$
\lambda_{1,2}=0.25 \pm 0.52 i, \quad \lambda_{3}=-0.6 .
$$

In this case system have one real negative eigenvalue and one complex-conjugate pair of positive eigenvalues. This type of geometry is called saddle-focus.

\subsection{Lyapunov exponents}

It is known, that Lyapunov exponents provide a quantitative measurements of the divergence or convergence of nearby trajectories for the dynamical system. If we consider a small space of initial conditions in the phase space, for sufficiently short time scales, the effect of the dynamics will be to distort this set into a hyperellipsoid, stretched along some directions and contracted along others [34]. The spectrum of the Lyapunov exponents is defined in the form

$$
\begin{aligned}
L_{e x}\left(\mathbf{x}_{0}, \mathbf{y}_{0} \in T \mathbf{x}(t) R^{3}\right) & =\lim _{t \rightarrow \infty} \frac{1}{t} \frac{\left\|D_{x} \Phi\left(t, \mathbf{x}_{0}\right) \mathbf{y}_{0}\right\|}{\left\|\mathbf{y}_{0}\right\|} \\
& =\lim _{t \rightarrow \infty} \frac{1}{t} \ln \left\|D_{x} \Phi\left(t, \mathbf{x}_{0}\right) \mathbf{y}_{0}\right\|,
\end{aligned}
$$

where $T \mathbf{x}(t)$ is a tangent space in the point on the fiducial trajectory and $\boldsymbol{y}(t)=D_{x} \Phi\left(t, \mathbf{x}_{0}\right) \boldsymbol{y}_{0}$ is solution of the linearized system [34]. The usual test for chaos is calculation of the largest Lyapunov exponent $\left(L E_{\max }\right)$ and a positive value indicates chaos [31]. It also determines a notion of predictability for a dynamical system. Figure 5 shows convergence plot of the $L E_{\max }(\alpha=0.6)$ and numerical values are following

$$
\begin{gathered}
L E_{\max 1}=0.0276, \quad L E_{\max 2}=0.0006 \\
L E_{\max 3}=-0.584
\end{gathered}
$$

\subsection{Bifurcation diagram}

A bifurcation is defined as a qualitative change in the dynamical behavior of the system of its phase portrait as one or more parameters are changed. Any point in the parameter set, where the behavior of dynamical system is unstable is called a bifurcation point, and the set of these points is called a bifurcation set [33]. This set can contain infinite number of the points but usually has zero measure [31]. Figure 6 shows bifurcation diagram generated by (1). We choosed the parameter $\alpha$ as the bifurcation parameter in the range $0.01 \leq \alpha \leq 0.6$. Bifurcation diagram shows that there exists many real numbers $\alpha$ for which is system solution chaotic.

\section{CIRCUITRY REALIZATION}

The circuit design procedure is based on classical circuit synthesis and the proposed circuit works in mixed mode [35-40].An advantage of this implementation is evident in comparison with older publication [6]: a smaller number of passive and active circuit elements. Operational transconductance amplifier OPA860 [41] and multiple output transconductance amplifier MAX435 [42] are used for circuitry implementation of the mathematical model equations (1).Nonlinearities are formed by a connection of four-quadrant analog multipliers AD633 [44]. 


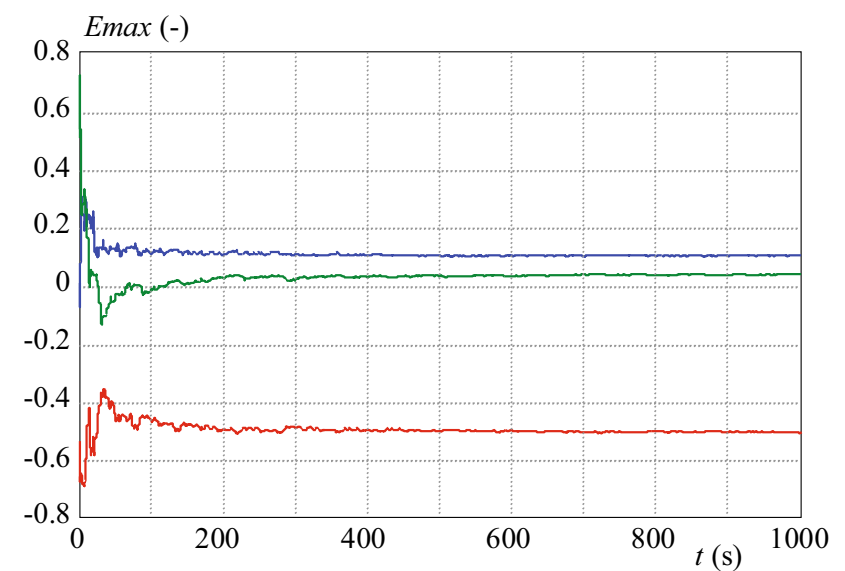

Fig. 5. Convergence plot of the largest Lyapunov exponents determined by $(1) ; \alpha=0.6$

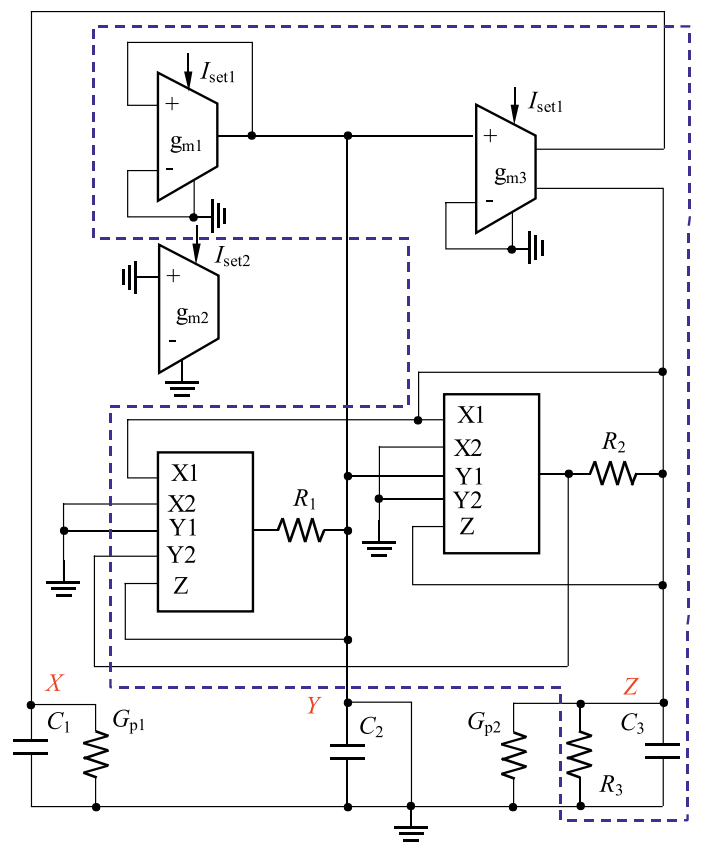

Fig. 7. Circuit realization of the chaotic system with OTA (OPA860), MOTA (MAX435) and analog multiplier (AD633) based on (1)), capacitors are $470 \mathrm{nF}$ and resistors are $R_{1}=15 \Omega, R_{2}=$ $100 \Omega$. Resistor $R_{3}$ should be adjustable from 0 to $1 k \Omega$

High (10 M $\Omega$ ) input resistances make signal source loading negligible. Therefore, we can straight connect input Y2 of the first multiplier to the output $\mathrm{W}$ of the second multiplier. We used this components for practical verification of a function, especially MAX435. We can use two OPA860 as replacement of MAX435 and up to date alternative. The schematic of the chaotic oscillator is shown in Fig. 7. Values of used passive elements were choosen $C_{1}=C_{2}=C_{3}=470 \mathrm{nF}, R_{1}=15 \Omega, R_{2}=100 \Omega$ and $R_{3}=600 \Omega$ (variable). We used the following simplifications: $g_{m 1}=\frac{1}{3} \mathrm{mS}, I_{S E T 1}=15.2 \mathrm{~m} \mathrm{~A}, g_{m 2}=\frac{1}{2} \mathrm{mS}$, $I_{S E T 2}=15.2 \mathrm{~mA} g_{m 3}=1 \mathrm{mS}, I_{S E T 3}=850 \mu \mathrm{A}$. Circuit is powered by symmetrical voltages $\pm 5 \mathrm{~V}$ (OTA and

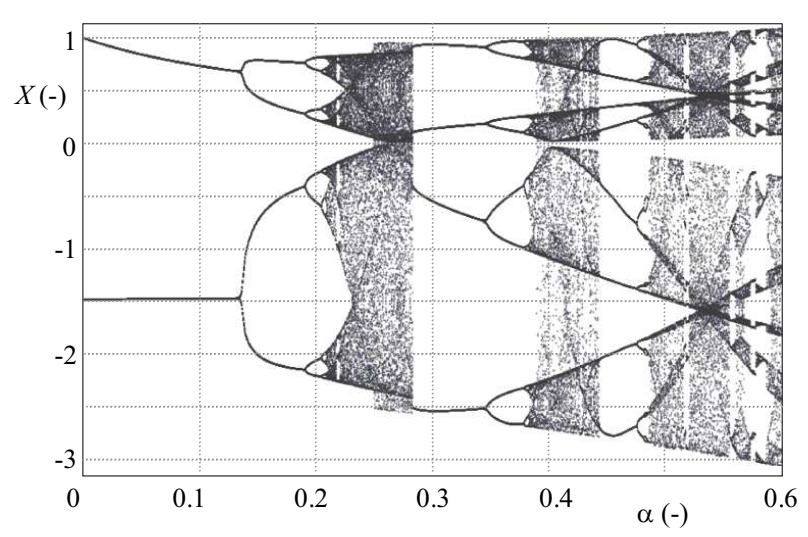

Fig. 6. Bifurcation diagram generated by (1), the bifurcation parameter $\alpha$ is shown on the horizontal axis of the plot

MOTA) resp. $\pm 15 \mathrm{~V}$ (AD633).

$$
\begin{aligned}
-\left(C_{1}+C_{p 1}\right) \frac{\mathrm{d} u_{1}}{\mathrm{~d} t} & =G_{p 1} u_{1}-g_{m 3} u_{2} \\
-\left(C_{2}+C_{p 2}\right) \frac{\mathrm{d} u_{2}}{\mathrm{~d} t} & =\frac{1}{3} g_{m 2} u_{1}-\left(\frac{1}{2} g_{m 1}-G_{p 2}\right) u_{2} \\
& +\frac{1}{2} u_{2} u_{3}^{2} \\
-\left(C_{3}+C_{p 3}\right) \frac{\mathrm{d} u_{3}}{\mathrm{~d} t} G_{p 2} & =g_{m 3} u_{2}+\left(G+G_{p 3}\right) u_{3}-u_{2} u_{3}
\end{aligned}
$$

\section{INFLUENCE OF PARASITIC PROPERTIES OF ACTIVE ELEMENTS}

Non-ideal active elements are depicted in Figs. 8 and 9. Parasitic analysis deals mainly with input and output properties of used active element that cause significant problems in the state space. Important parasitic admittances of the circuit (signed as $Y_{p}$ ) are caused by the real input and output properties of used active elements and are shown in Fig. 7. Common input and output small signal parameters for OTA are $R_{\text {in_OTA }}=455 \mathrm{k} \Omega$, $R_{\text {out_OTA }}=54 \mathrm{k} \Omega, C_{\text {in_OTA }}=2.2 \mathrm{pF}, C_{\text {out_OTA }}=2 \mathrm{pF}$ and for MOTA are $R_{\text {in_OTA }}=800 \mathrm{k} \Omega, R_{\text {out_OTA }}=$ $3.5 \mathrm{k} \Omega, C_{\text {in_ota }}=4 \mathrm{pF}, C_{\text {out_OTA }_{1}}=4.1 \mathrm{pF}$.

We suppose three locations (input and output admittances in three nodes) where is the highest impact of parasitic properties. These parasitic admittances (see Fig. 7) can be expressed as

$$
\begin{aligned}
& Y_{p 1}=G_{p 1}+s C_{p 1}=\left(G_{\text {pin } 2}+G_{\text {pout } 3}\right)+s\left(C_{\text {pin } 1}+C_{\text {pout } 3}\right)= \\
& \frac{1}{R_{\text {in_OTA }_{2}}}+\frac{1}{R_{\text {Out_MOTA }}}+s\left(C_{\text {in_OTA }_{1}}+C_{\text {out_MOTA }}\right) \text {, } \\
& Y_{p 2}=G_{p 2}+s C_{p 2}=\left(G_{\text {pin } 1}+G_{\text {pin } 3}+G_{\text {pout } 1}+G_{\text {pout } 2}\right) \\
& +s\left(C_{\mathrm{pin} 1}+C_{\mathrm{pin} 3}+C_{\mathrm{pout} 1}+C_{\mathrm{pout} 2}\right)= \\
& =\frac{1}{R_{\text {in_OTA }_{1}}}+\frac{1}{R_{\text {in_MOTA }_{2}}}+\frac{1}{R_{\text {out_OTA }_{1}}}+\frac{1}{R_{\text {out_OTA }_{2}}}+ \\
& s\left(C_{\text {in_OTA }_{1}}+C_{\text {in_MOTA }}+C_{\text {out_OTA }_{1}}+C_{\text {out_MOTA }}\right)
\end{aligned}
$$




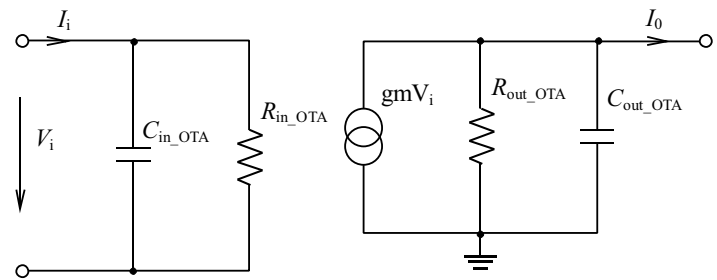

Fig. 8. Convergence plot of the largest Lyapunov exponents determined by (1); $\alpha=0.6$

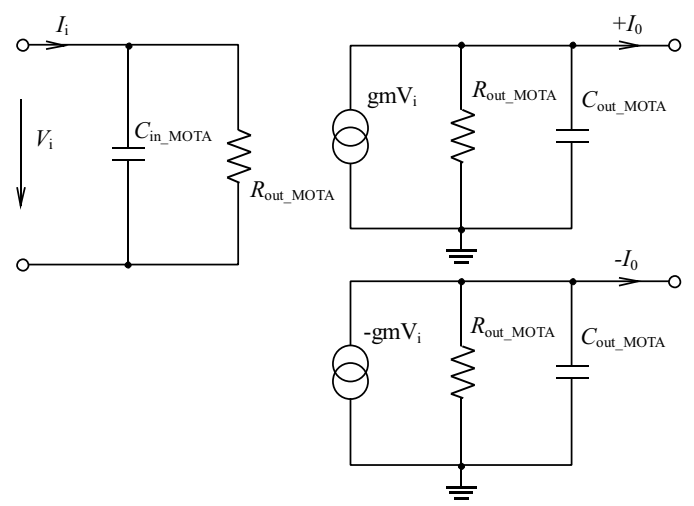

Fig. 9. Bifurcation diagram generated by (1). The bifurcation parameter $\alpha$ is shown on the horizontal axis of the plot

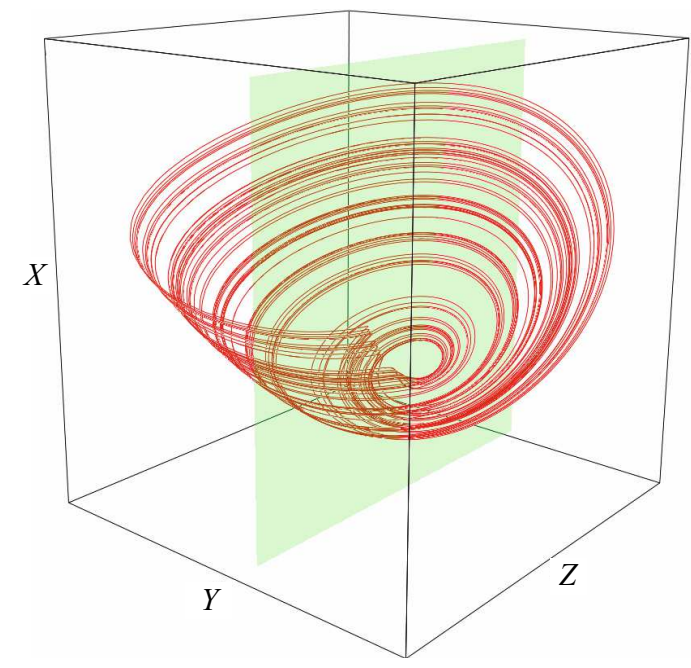

Fig. 10. Simulation in PSpice with indication of the $x-z$ plane sliced at $y=0$ (green surface)

$Y_{p 3}=G_{p 3}+s C_{p 3}=G_{p o u t 3}+s C_{p o u t 3}=$

$$
\frac{1}{R_{\text {out_MOTA }}}+s C_{\text {out_MOTA }},
$$

Now we will consider the new form of the system (1), where state matrix $A_{p}$ is represented influence of the parasitic admittances of the active elements.

$$
\begin{gathered}
\dot{\mathbf{x}}=\left(\mathbf{A}+\mathbf{A}_{p}\right) \mathbf{x}+\mathbf{B} \mathbf{x} \mathbf{x}^{T} \mathbf{C}+\mathbf{D} \mathbf{x}\left(\mathbf{x}^{T} \mathbf{C}\right)^{2}, \\
\mathbf{A}=\left(\begin{array}{ccc}
0 & g_{m 3} & 0 \\
-\frac{1}{3} \cdot g_{m 2} & \frac{1}{2} \cdot g_{m 1} & 0 \\
0 & -g_{m 3} & -0.6
\end{array}\right),
\end{gathered}
$$

$$
\begin{gathered}
\mathbf{A}_{p}=\left(\begin{array}{ccc}
-G_{p 1} & 0 & 0 \\
0 & -G_{p 2} & 0 \\
0 & 0 & -G_{p 3}
\end{array}\right), \\
\mathbf{B}=\left(\begin{array}{lll}
0 & 0 & 0 \\
0 & 0 & 0 \\
0 & 1 & 0
\end{array}\right), \quad \mathbf{C}=\left(\begin{array}{l}
0 \\
0 \\
1
\end{array}\right) \\
\mathbf{D}=\left(\begin{array}{ccc}
0 & 0 & 0 \\
0 & -\frac{1}{2} & 0 \\
0 & 0 & 0
\end{array}\right)
\end{gathered}
$$

The Jacobian matrix and the local behavior of the system (12) near the origin with influence of parasitic properties of active elements is

$$
\mathbf{J}_{p}=\left(\begin{array}{ccc}
-G_{p 1} & 1 & 0 \\
-\frac{1}{3} & -\frac{1}{2} z^{2}+\frac{1}{2}-G_{p 2} & -y z \\
0 & z-1 & y-0.6-G_{p 3}
\end{array}\right)
$$

and characteristic polynomial for critical point $(0,0,0)$ is

$$
\operatorname{det}\left(\lambda \mathbf{I}-\mathbf{J}_{p}\right)=\lambda^{3}+1.714 \lambda^{2}+1.223 \lambda+0.433=0 .
$$

Now, we get a new values of eigenvalues

$$
\lambda_{4,5}=0.086 \pm 0.44 i \quad \lambda_{6}=-0.886
$$

\section{SIMULATION AND MEASUREMENT RESULTS}

The circuitry implementation functionality was first successfully tested in PSpice simulator. Figure 10 resp. Figs. 11 to 14 show simulation results. Figure 10 was obtained by data export from the PSpice to the MathCAD environment and was processed to the $3 \mathrm{D}$ plot. Correct function of the dynamical system was also verified experimentally on the breadboard. Plane projections of the selected signals were measured by means of an oscilloscope HB 54603B. Figure 21 shows a photo of the measurement results - projection of chaotic attractor onto $x-y$ plane. Comparison of results proved a rather good agreement between numerical simulation, PSpice simulation and measurement.

\section{CONCLUSION}

In this work, we reported how to build the autonomous chaotic circuit based on memristive element. The suitability of OTAs as the main active element to obtain basic building block for the design of nonlinear circuit was verified and it was previously published in many other publications. Nevertheless, very important is thinking to influence of parasitic properties of active elements. The crux of the paper is an analog circuit realization of the 

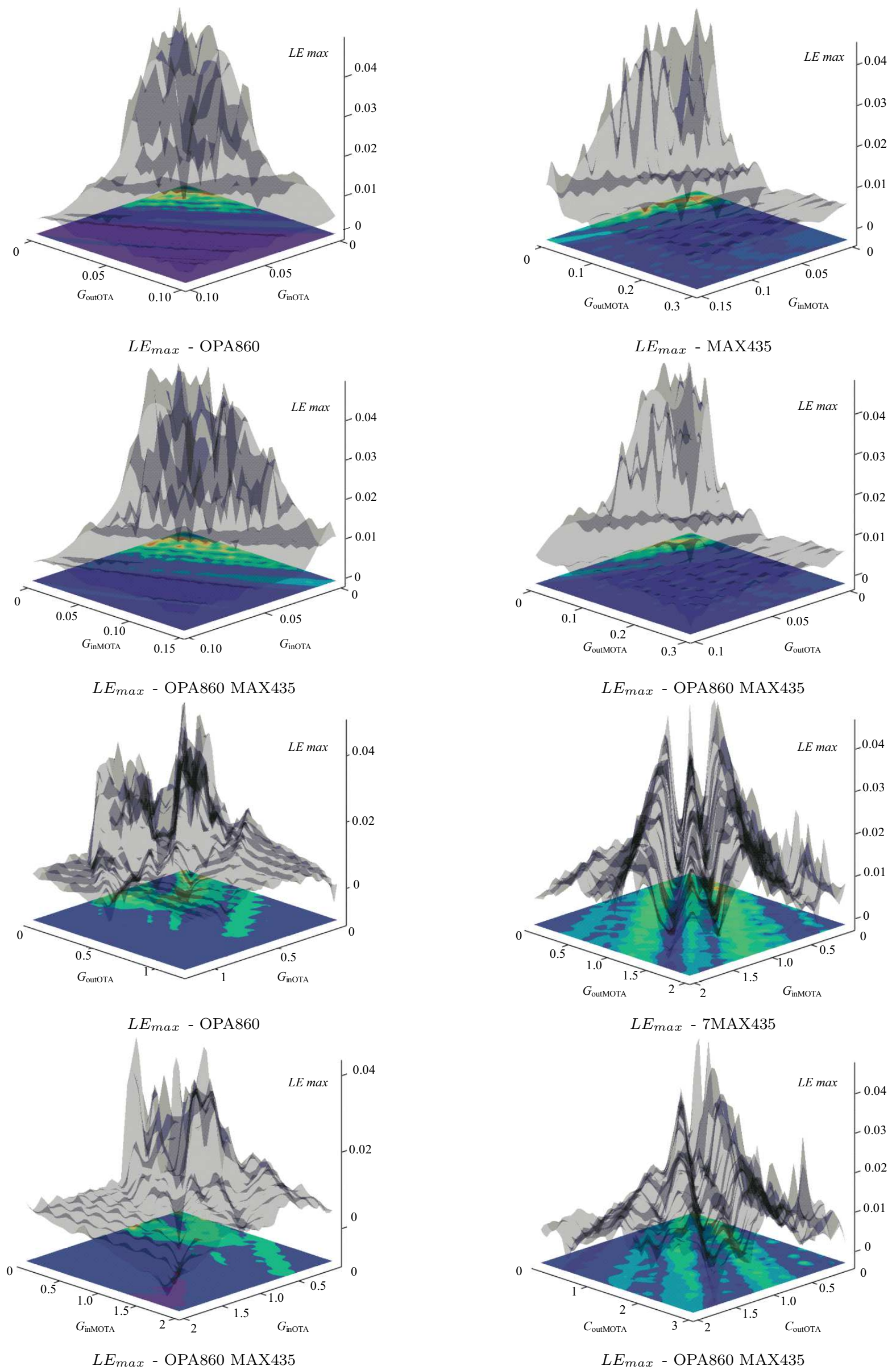

Fig. 11. Infuence of parasitic admittances on the size of the largest Lyapunov exponent 


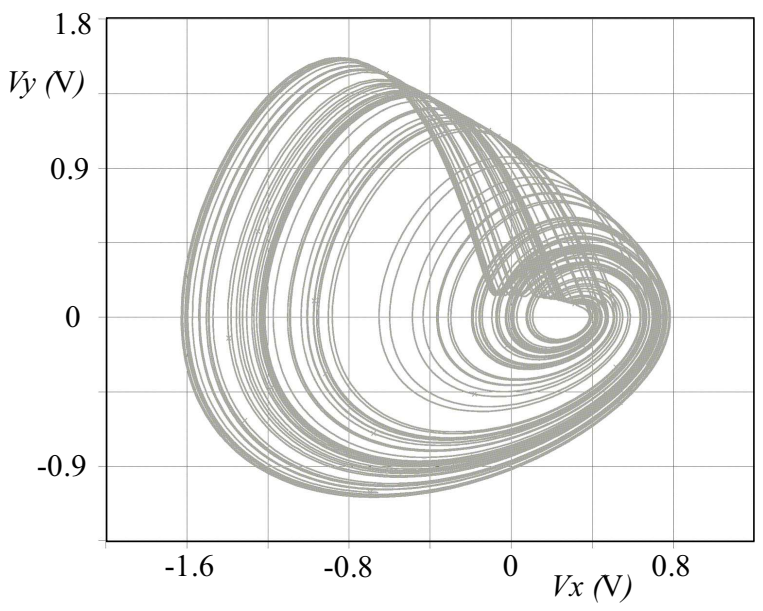

Fig. 12. Plot of $v_{x}(t)$ versus $v_{y}(t)$ plane projection of the chaotic attractor - PSpice simulation

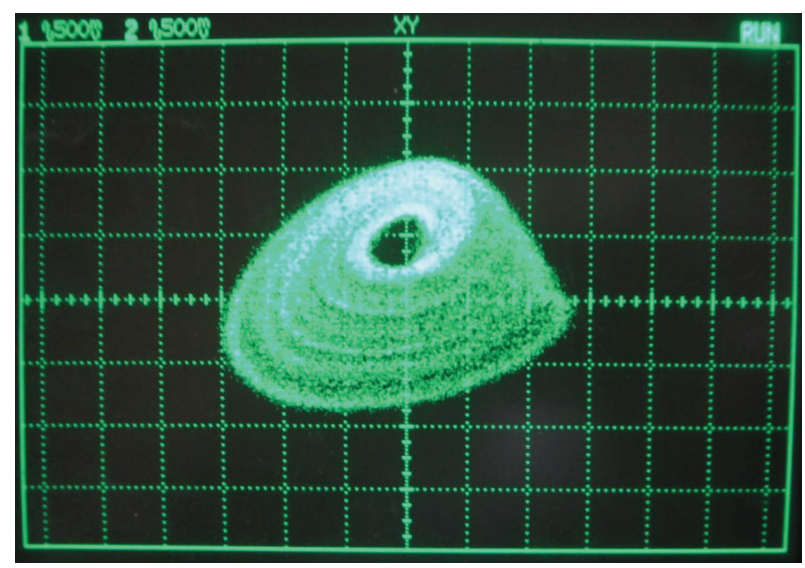

Fig. 14. The $v_{x}(t)$ versus $v_{y}(t)$ plane projection of the chaotic attractor - measurement results. The axes scales for the experimental attractor are on the both axes $500 \mathrm{mV} / \mathrm{div}$

memristor based chaotic circuit via operational transconductance amplifiers, analog multipliers and without coil. Simplification of the circuit is also evident in comparison with realization by Muthuswamy and Chua [6]. We have started with obtain of the chaotic attractors by mathematical analysis. In other part is presented circuit realization with the theoretical PSpice simulation and the practical measurement results on breadboard are presented at the end.

Other crux of this article is in simulations and calculations of eigenvalues with respect to influence of parasitic properties of active elements. It appears, that some influences of the parasitic properties are crucial. In our proposed circuit we discovered that the most critical to chaos destruction seems to be parasitic output resistance of the MOTA element MAX435. This study raises the question whether we are able to compensate these undesirable effects. It seems that we can found solution in the change of some parameter of active elements.

Based on the results from this paper, there are a plethora of opportunity for a future work in this area. Certainly, there are a plethora many similar circuitry implementation of memristor based chaotic circuits, as we

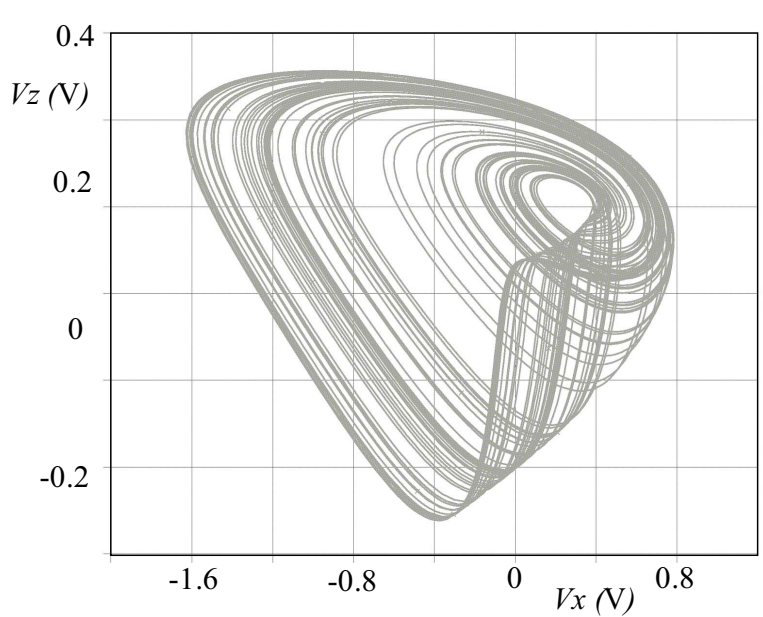

Fig. 13. Plot of $v_{x}(t)$ versus $v_{z}(t)$ plane projection of the chaotic attractor - PSpice simulation

were also mentioned in this article. Nevertheless, with a technological developments it is still relevant deals with new possibilities of the circuit realization and mainly with simplification of the designed circuit structure. Also possibility of implementation in the RF area seems to be very interesting [27], [28].

\section{Acknowledgements}

The support of the project CZ.1.07/2.3.00/20.0007 WICOMT, financed from the operational program Education for competitiveness, is gratefully acknowledged. The described research was performed in laboratories supported by the SIX project; the registration number CZ.1.05/2.1.00/03.0072, the operational program Research and Development for Innovation. The research is also part of the specific research grant denoted FEKT-S$11-13$.

\section{REFERENCES}

[1] CHUA, L. O. : Memristor - The Missing Circuit Element, IEEE Transaction Circuit Theory 18 No. 5 (1971), 507-519.

[2] CHUA, L. O.-KANG, S. M. : Memristive Devices and Systems, Proceedings of the IEEE 64 No. 2 (1976), 209-223.

[3] STRUKOV, D. B.-SNIDER, G. S.-STEWART, G. R.-WILLIAMS, R. S.: The Missing Memristor Found, Nature 453 (2008), 80-83.

[4] ITOH, M.-CHUA, L. O. : Memristor Oscillators., International Journal of Bifurcation and Chaos 18 No. 11 (2008), 3183-3206.

[5] MUthuswamy, B. : Implementing Memristor Based Chaotic Circuits, International Journal of Bifurcation and Chaos 20 No. 5 (2010), 1335-1350.

[6] Muthuswamy, B.-CHUA, L. O.: Simplest Chaotic Circuit, International Journal of Bifurcation and Chaos 20 No. 5 (2010), 1567-1580.

[7] Muthuswamy, B.-KOKATE, P. P.: Memristor-Based Chaotic Circuits, IETE Technical Review 26 No. 6 (2009), 415-426.

[8] BAO, B. C.-LIU, Z.-XU, J. P.: Steady Periodic Memristor Oscillator with Transient Chaotic Behaviours, Electronics Letters 46 No. 3 (2010), 237-238. 
[9] WANG, W.-WANG, W.-TAN, D. : A New Memristor Based Chaotic Circuit, Fourth International Workshop on Chaos-Fractals Theories and Applications, 2011, pp. 57-60.

[10] QI, A.-WANG, G.: Chaotic Oscillator Based on Memristor and its Circuit Implementation, Fourth International Workshop on Chaos-Fractals Theories and Applications, 2011, pp. 328-331.

[11] WEIHENG, S.-CHUNFU, L.-JUEBANG, Y. A. : Memristor Based Chaotic Oscillator, International Conference on Communications, Circuits and Systems, 2009, pp. 955-957.

[12] ZHANG, J.-ZHANG, H.-ZHANG, G.: Controlling Chaos in a Memristor-Based Chua's Circuit, International Conference on Communications, Circuits and Systems, 2009, pp. 961-963.

[13] YU, Q.- QIN, Z.-YU, J.-MAO, Y.: Realization of an Analog Model of Memristor based on Light Dependent Resistor, International Conference on Communications, Circuits and Systems, 2009, pp. 974-977.

[14] IU, H. H. C.-YU, D. S.-FITCH, A. L.-SREERAM, V.: Chaos Control in a Memristor Based Circuit, IEEE International Symposium onCircuits and Systems, 2011, pp. 2946-2949.

[15] VALSA, J.-BIOLEK, D.-BIOLEK, Z. : An Analogue Model of the Memristor, International Journal of Numerical Modeling: Electronic Networks, Device and Fields 24 (2011), 400-408.

[16] BIOLEK, Z.-BIOLEK, D.-BOLKOVA, V. : SPICE Model of Memristor with Nonlinear Dopant Drift, Radioengineering 18 No. 2 (2009), 210-214.

[17] PETRAS, I.: Fractional-Order Memristor-Based Chua's Circuit, IEEE Transactions on Circuits and Systems I: Regular Papers 58 No. 6 (2011), 1323-1336.

[18] YENER, S.-KUNTMAN, H. : A New CMOS Based Memristor Implementation, International Conference on Applied Electronics, 2012, pp. 345-348.

[19] CORINTO, F.-ASCOLI, A.-GILLI, M. : Nonlinear Dynamics of Memristor Oscillators, IEEE Transactions on Circuits and Systems II: Express Briefs 57 No. 12 (2010,), 975-979.

[20] FITCH, A. L.-IU, H. H. C.-WANG, X. Y.-SREERAM, V.-QI, W. G. : Realization of an Analog Model of Memristor Based on Light Dependent Rresistor, IEEE International Symposium on Circuits and Systems, 2012, pp. 1139-1142.

[21] DRISCOLL, T.-PERSHIN, Y. V.-BASOV, D. N.-VENTRA, M.: Chaotic Memristor, Applied Physics A 102 No. 4 (2011), 885-889.

[22] NAKAGAWA, S.-SAITO, T.: An RC OTA Hysteresis Chaos Generator, IEEE Transactions on Circuits and Systems I: Fundamental Theory and Applications 45 No. 2 (1998), 182-186.

[23] NAKAGAWA, S.-SAITO, T.: Design and Control of RC VCCS 3-D Hysteresis Chaos Generators, IEEE Transactions on Circuits and Systems I: Fundamental Theory and Applications 43 No. 12 (1996), 1019-1021.

[24] ACHO, L.-VIDAL, Y.: Hysteresis Modeling of a Class of RC-OTA Hysteretic-Chaotic Generators, PHYSCON 2011, Leon, Spain, Sep 2011.

[25] SANCHEZ-SINENCIO, E.-RAMIREZ-ANGULO, J.-LINARES-BARRANCO, B.-RODRIGUEZ-VAZQUEZ, A. : Operational Transconductance Amplifier-Based Nonlinear Function Syntheses, IEEE Journal of Solid-State Circuits 24 No. 6 (Dec 1989), 1576-1586.

[26] SOTNER, R.-JERABEK, J.-DOSTAL, T.-VRBA, K. : Multifunctional Adjustable Current Mode Biquads Based on Distributed Feedback Voltage Mode Prototype with OTAs., International Journal of Electronics 97 No. 7 (July 2010), 797-809.

[27] GARCIA-LOPEZ, J. H.-JAIMES-REATEGUI, R.-PISARCHIK, A. N.-MURGUIA-HERNANDEZ, A.-MEDINA-GUTIERREZ, C.-VALDIVIA-HERNADEZ, R.-VILLAFANARAUDA, E. : Novel Communication Scheme Based on Chaotic Rössler circuits, International Conference on Control and Synchronzation of Dynamical Systems, 2005, pp. 276-284.
[28] RIAZ, A.-ALI, M.: Chaotic Communications, their Applications and Advantages over Traditional Methods of Communication, 6th International Symposium on Communication Systems, Networks and Digital Signal Processing, 2008, pp. 21-24.

29] ThOmpson, J. M. T.-STEWART, H.-B.: Nonlinear Dynamics and Chaos, $2^{\text {nd }}$ edition, Wiley, 2002.

30] SPROTT, J. C.-LINZ, S. J.: Algebraically Simple Chaotic Flow, International Journal of Chaos Theory and Applications 5 No. 2 (2000), 1-20.

31] SPROTT, J. C.: Chaos and Time-Series Analysis, Oxford University Press, 2003.

[32] HIRSCH, M. W.-SMALE, S. : Differential Equations, Dynamical Systems and Linear Algebra, Academic Press, New York, 1974.

33] PATIDAR, V.-SUD, K. K.: Bifurcation and Chaos in Simple Jerk Dynamical Systems, PRAMANA-journal of physics 64 No. 4 (Jan 2005), 75-93.

[34] SANDRI, M. : Numerical Calculation of Lyapunov Exponents, The Mathematica Journal 6 No. 3 (1996), 78-84.

35] PETRZELA, J.-KOLKA, Z.-HANUS, S.: Simple Chaotic Oscillator: From Mathematical Model to Practical Eexperiment, Radioengineering 15 No. 1 (2006), 6-12.

36] PETRZELA, J.-DRINOVSKY, J.: High Frequency Chaos Converters, In Proceedings IEEE Region 8 International Conference on Computational Technologies in Electrical and Electronics Engineering, SIBIRCON-2010, 2010, pp. 750-754.

[37] PETRZELA, J.: On the Piecewise-Linear Approximation of the Polynomial Chaotic Dynamics, In $34^{\text {th }}$ International Conference on Telecommunications and Signal Processing, 2011, pp. 319-323.

[38] Petrzela, J.-GotThans, T.: Chaotic Oscillators with Single Polynomial Nonlinearity and Digital Sampled Dynamics, Przeglad Elektrotechniczny 3 No. 1 (2011), 161-163.

[39] Petrzela, J.-Gotthans, T.-Hrubos, Z. : Modeling Deterministic Chaos Using Electronic Circuits, Radioengineering 20 No. 2 (2011), 438-444.

[40] PETRZELA, J.-HRUBOS, Z.: Simplest Chaos Converters: Modeling, Analysis and Future Perspectives, In Proceedings of the $8^{\text {th }}$ WSEAS International Conference on System Science and Simulation in Engineering, 2009, pp. 160-163.

[41] Texas Instruments. OPA860: Wide Bandwidth Operational Transconductance Amplifier (OTA) and Buffer OPA860 (datasheet) Cited 2012-02-20. Available at: http://focus.ti.com/lit/ds/symlink/opa860.pdf.

[42] Maxim. MAX435: Wideband Transconductance Amplifier (datasheet) Cited 2012-02-20. Available at: http://datasheets.maxim-ic.com/en/ds/MAX435-MAX436.pdf.

[43] Analog Devices. AD633: Low Cost Analog Multiplier (datasheet) Cited 2012-02-20. Available at: http://www.analog.com.

Received 12 May 2013

Zdeněk Hruboš was born in Uherské Hradiště, Czech Republic, in 1984. He received his MSc degree in 2009 from Brno University of Technology, Czech Republic. He is currently studying his $\mathrm{PhD}$ study at the same university. His research interest is in computer analysis and synthesis of electronics circuits and nonlinear systems.

Tomáš Gotthans was born in Brno, Czech Republic, in 1985. He received the MSc degree at the Brno University of Technology in 2010. Now he is a PhD student at the Department of Radio Electronics, Brno University of Technology. His research interests include programming, microprocessors and mixed-mode circuit synthesis. 